\title{
PROPRIÉTÉ COOPÉRATIVE ET ACCEPTABILITÉ SOCIALE DE L'ÉOLIEN TERRESTRE
}

Thomas Bauwens

De Boeck Supérieur | «Reflets et perspectives de la vie économique »

2015/1 Tome LIV | pages 59 à 70

ISSN 0034-2971

ISBN 9782807301283

Article disponible en ligne à l'adresse :

http://www.cairn.info/revue-reflets-et-perspectives-de-la-vie-economique-2015-1-page-59.htm

\section{!Pour citer cet article :}

Thomas Bauwens, «Propriété coopérative et acceptabilité sociale de l'éolien terrestre », Reflets et perspectives de la vie économique 2015/1 (Tome LIV), p. 59-70.

DOI 10.3917/rpve.541.0059

Distribution électronique Cairn.info pour De Boeck Supérieur.

(C) De Boeck Supérieur. Tous droits réservés pour tous pays.

La reproduction ou représentation de cet article, notamment par photocopie, n'est autorisée que dans les limites des conditions générales d'utilisation du site ou, le cas échéant, des conditions générales de la licence souscrite par votre établissement. Toute autre reproduction ou représentation, en tout ou partie, sous quelque forme et de quelque manière que ce soit, est interdite sauf accord préalable et écrit de l'éditeur, en dehors des cas prévus par la législation en vigueur en France. Il est précisé que son stockage dans une base de données est également interdit. 


\title{
Propriété coopérative et acceptabilité sociale de l'éolien terrestre
}

\author{
Thomas Bauwens ${ }^{1}$
}

\begin{abstract}
Résumé - Le développement éolien terrestre en Belgique se heurte régulièrement à des contestations locales. Face à ce problème, les coopératives éoliennes semblent présenter des atouts essentiels. Cet article explique, dans un premier temps, pourquoi ces organisations sont susceptibles de renforcer l'acceptabilité sociale de cette technologie. Dans un second temps, il confronte ces hypothèses normatives à la réalité de données empiriques. Les résultats de cette analyse confirment l'effet positif de la propriété coopérative sur l'acceptabilité sociale de l'éolien terrestre.
\end{abstract}

Mots clés: Éolien, énergie renouvelable, coopérative, acceptabilité sociale, participation, NIMBY.

\begin{abstract}
Cooperative Ownership and Social Acceptability of Onshore Wind Power

Onshore wind power development in Belgium has provoked considerable opposition at the local level. To cope with this issue, wind power cooperatives seem to present important assets. This article explores the reasons why these organizations are likely to enhance social acceptability of this technology. Then it confronts these normative assumptions with empirical data. The results of this analysis confirm the positive effect of cooperative ownership on the social acceptability of onshore wind turbines.
\end{abstract}

Codes JEL : L31, P13, Q42

\section{INTRODUCTION}

La nécessité d'un déploiement accru des énergies renouvelables est largement acceptée comme l'un des piliers de la transition vers un secteur énergétique

1. Thomas Bauwens est doctorant en sciences économiques au Centre d'économie sociale, HEC École de gestion de l'Université de Liège. Depuis mai 2014, il est aussi chercheur invité au Environmental Change Institute, Université d'Oxford. 
soutenable. Dans cette perspective et conformément aux directives européennes, la Belgique compte, à l'horizon 2020, accroître sensiblement la part des énergies renouvelables dans sa consommation finale d'énergie, qui devrait atteindre $13 \%$. Parallèlement à cet objectif national, chaque région a également établi ses propres quotas. Ainsi, le gouvernement wallon projetait, jusqu'en avril 2015, d'atteindre une production de 8000 GWh d'électricité renouvelable par an en 2020. Près de la moitié de celle-ci devait être produite par l'éolien terrestre (3 800 GWh), alors que la capacité installée actuelle produit environ 1354 GWh sur base annuelle ${ }^{2}$. En Flandre, les autorités ont prévu d'accroître la capacité de l'éolien terrestre, qui représente actuellement $603 \mathrm{MW}$, pour atteindre $1063 \mathrm{MW}$. Malgré une opinion publique favorable (IPSOS-Belgium 2010), cette technologie soulève cependant de nombreuses résistances locales de la part des résidents et des communes, dans un contexte de densité démographique élevée. À tel point que cette opposition pourrait compromettre les objectifs prévus en la matière. Le but de cet article est d'évaluer, à partir d'une analyse économétrique quasi expérimentale, le rôle que peut jouer la propriété coopérative des éoliennes pour résoudre ce problème.

\section{CONTEXTE : LE SECTEUR ÉOLIEN TERRESTRE EN BELGIQUE}

Le graphique 1 présente l'évolution de la puissance installée de l'éolien terrestre en Flandre et en Wallonie de 1997 à 2014. Au niveau national, la capacité installée cumulée (courbe continue sur le graphique) a crû exponentiellement entre 2002 et 2011, passant de 31,4 MW à 885,1 MW. Les premiers projets ont essentiellement été réalisés en Flandre, mais la Wallonie a rapidement emboîté le pas et a connu une expansion beaucoup plus rapide après 2006 . Cependant, cette croissance s'est considérablement affaiblie depuis 2011, surtout dans le Sud du pays. En 2014, alors que le secteur a connu une recrudescence en Flandre, ce dernier stagnait en Wallonie, avec 9 éoliennes installées durant l'année. Ce ralentissement est principalement dû au nombre croissant d'appels juridiques introduits contre les projets éoliens (Apere, 2014). En décembre 2014, 37 projets, qui représentaient 215 éoliennes et une capacité de 592 MW, étaient en procédure de recours en Wallonie.

Par ailleurs, le contexte institutionnel belge est peu favorable à la participation citoyenne. Le secteur éolien s'est principalement développé selon un modèle "top-down », tandis que les initiatives participatives demeurent une exception (Pepermans et Loots, 2013). Celles-ci sont notamment obérées par la logique du « premier arrivé, premier servi » qui prévaut lors de la recherche d'emplacements pour les parcs éoliens. Selon ce système, les autorités traitent les requêtes de permis dans un ordre chronologique. Cette politique,

2. Au moment de l'écriture de cet article, le gouvernement wallon a revu ces objectifs à la baisse. ॥ est maintenant prévu d'atteindre une production de 8000 GWh d'électricité renouvelable par an en 2026. 
conjuguée à la rareté des zones favorables et à leur délimitation par les autorités publiques, ainsi que le nombre croissant de développeurs éoliens, ont concouru à la création d'un environnement hautement compétitif et ont encouragé un véritable " wind rush », c'est-à-dire une ruée des développeurs sur les sites disponibles. Ce phénomène entrave sévèrement les possibilités d'interactions avec les riverains et force les développeurs à impliquer la population après que le projet soit déjà défini, car ils courent le risque de perdre leurs sites au profit d'un concurrent. Or l'acceptabilité d'un parc éolien implique un processus relativement long et devrait donc débuter le plus tôt possible dans la mise en place d'un projet (Pepermans et Loots, 2013). Enfin, ce contexte compétitif tend à défavoriser les petits acteurs, tels que les coopératives, qui manquent souvent de temps et de ressources pour agir aussi rapidement que les grands développeurs.

\section{Graphique 1. Capacité installée de l'éolien terrestre en Belgique,} 1997-2014

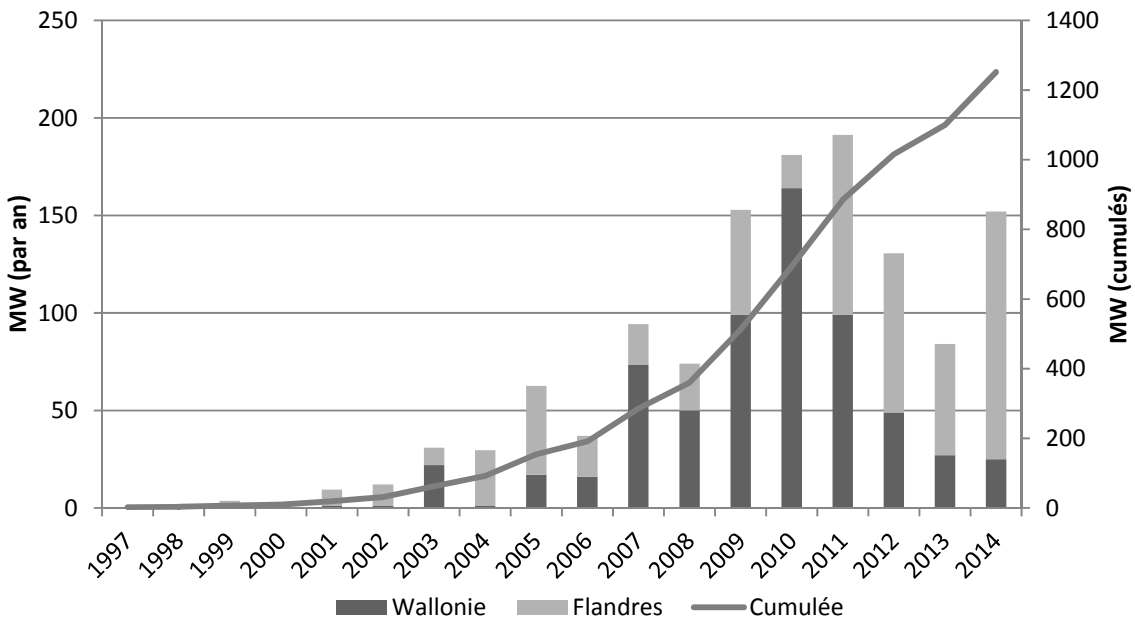

Source : créé par l'auteur sur base de données de l'APERE et ODE Vlaanderen.

Le nouveau cadre de référence wallon, adopté en 2013, semble vouloir corriger cette situation, puisqu'il oblige les développeurs éoliens à ouvrir leur capital à hauteur de 24,99 \% pour la participation citoyenne et 24,99\% pour celle des communes. Pareille disposition n'existe pas encore dans le Nord du pays. Cependant, cette initiative n'a pas pu, jusqu'à présent, contrer les effets pervers de la logique qui caractérise actuellement le processus de planification. En effet, comme le montre le graphique 2, la part détenue par les collectivités locales est actuellement limitée à $8 \%$. En Flandre, les deux principales coopératives représentaient en 2013 environ 4 \% de la capacité éolienne installée. 


\section{Graphique 2. Répartition des développeurs éoliens selon la forme de propriété en Wallonie}

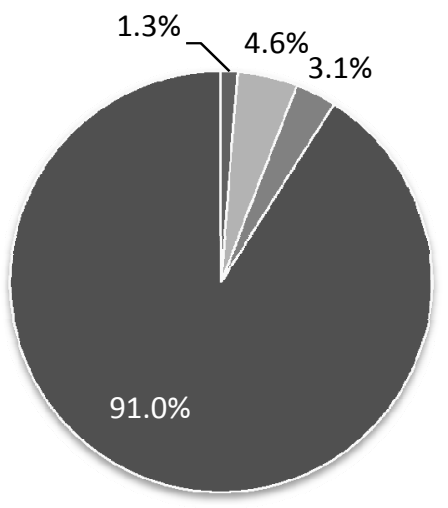

- Communes

- Coopératives

- Inter-communales

- Entreprises privées

Source : Apere (2015).

\section{PROBLÉMATIQUE ET CADRE CONCEPTUEL}

\subsection{Une technologie contestée}

Le développement éolien terrestre suscite des mouvements d'opposition locale en maints endroits dans le monde. Les raisons invoquées par ses détracteurs résident principalement dans les impacts sur les paysages naturels et les effets négatifs sur le tourisme qui en résultent, la génération de pollution sonore ou d'effets stroboscopiques gênants, les conséquences négatives sur les prix des propriétés foncières et immobilières ainsi que les risques encourus par la faune et la flore locale. Dans de nombreux cas, cette résistance prend la forme de réseaux citoyens formalisés tels que Vent de Colère (France), Vent de Raison (Belgique), Opzione 0 (Italie), laeden (Espagne), Stilhed (Danemark), etc. La Belgique, où, comme nous l'avons vu, le ralentissement du développement des projets éoliens résulte en grande partie de l'hostilité à l'égard de cette technologie, n'échappe pas à ce phénomène.

\subsection{L'explication " NIMBY " et ses limites}

Cette hostilité locale aux projets éoliens est communément expliquée en termes d'effet "NIMBY » ("Not In My Back Yard »), qui désigne la position d'individus qui perçoivent l'énergie éolienne comme positive pour la société en général, mais dont l'analyse coût-bénéfice personnelle les pousse à s'opposer à la construction de parcs éoliens dans leur voisinage immédiat. D'un point de vue 
économique, l'effet NIMBY représente un dilemme social, c'est-à-dire une situation dans laquelle l'intérêt collectif entre en contradiction avec les stratégies individuelles (Frey et Oberholzer-Gee, 1997). En effet, l'énergie éolienne comporte des externalités positives globales (expansion de la capacité d'énergie renouvelable et réduction des émissions de gaz à effets de serre liés à la génération d'énergie conventionnelle, création d'emplois dans l'industrie de l'énergie renouvelable, diminution de la dépendance aux importations de ressources, etc.). Cependant, les riverains immédiats des projets n'ont pas d'incitant à coopérer à leur réalisation, puisque ces derniers leur imposent des coûts nets considérables. Si ce scénario se généralise dans toutes les localités, un problème d'action collective survient, menant à une fourniture sous-optimale de l'énergie éolienne.

II apparaît ainsi que l'explication NIMBY repose sur l'hypothèse selon laquelle les individus agissent de manière rationnelle et sont mus par leur intérêt propre, conformément à la théorie conventionnelle des dilemmes sociaux (Olson, 1965). Cependant, des études empiriques plus récentes montrent que les acteurs impliqués dans un dilemme social ne recherchent pas systématiquement à maximiser leurs bénéfices individuels. Ils attachent, par exemple, de l'importance à la manière dont les autres se comportent ainsi qu'aux valeurs, aux codes comportementaux et aux normes sociales en jeu (Ben-Ner et Putterman, 1999). En outre, il apparaît que les émotions jouent un rôle crucial dans les décisions individuelles, qui sont donc loin d'être purement rationnelles. En ce qui concerne l'acceptabilité des projets éoliens, la littérature existante a mis en exergue le concept d'« attachement au lieu ", qui désigne un lien affectif et symbolique unissant les individus à leur lieu de vie (Devine-Wright, 2009 ; Cass et Walker, 2009). Dans cette perspective, le concept NIMBY a fait l'objet de nombreuses critiques, selon lesquelles celui-ci est trop simpliste et inapproprié pour appréhender les motivations réelles de la majorité des opposants (Wolsink, 2006 ; Devine-Wright, 2005).

\subsection{Par-delà le syndrome NIMBY}

Cherchant à dépasser le cadre NIMBY, plusieurs études ont montré que d'autres facteurs jouaient un rôle crucial dans l'acceptabilité des projets éoliens. Trois d'entre eux sont ici abordés plus en détail : la justice distributive, la justice procédurale et la confiance dans le développeur. La justice distributive désigne l'estimation individuelle subjective de la manière dont les coûts et les bénéfices sont distribués au sein d'un groupe. En l'occurrence, les bénéfices peuvent être monétaires, tels que les profits résultant de l'électricité générée et la création de nouveaux emplois dans la filière éolienne, ou non monétaires, comme des mesures compensatoires visant à réduire les impacts - environnementaux, sonores, ou autres - liés à l'implantation des mâts éoliens. De même, les coûts peuvent être liés à la dépréciation de la valeur des biens fonciers et immobiliers, à une modification du paysage local ou à la pollution sonore.

La justice procédurale, quant à elle, concerne la perception subjective d'équité dans le processus de mise en place des éoliennes et est liée à des aspects tels que le choix du site et la procédure de permis, les possibilités de participation, la quantité d'informations disponibles, etc. Les perceptions de justice et d'équité sont 
inhérentes au bien-être d'une communauté. Des situations perçues comme inéquitables peuvent mener à des protestations et à des conflits intracommunautaires, en particulier lorsque des décisions semblent avantager certains acteurs aux dépens d'autres parties. Ainsi, si les communautés locales perçoivent que des intérêts extérieurs monopolisent la plupart des bénéfices de l'énergie générée ou si elles ne sont pas impliquées dans les processus de développement, elles pourraient nourrir le sentiment d'être injustement traitées et prendre part à un activisme d'opposition.

Enfin, la confiance dans le développeur d'un projet éolien est cruciale lorsque son acceptabilité sociale est en jeu, surtout lorsque les riverains sont peu informés à propos de cette technologie. Elle permet l'établissement de relations de coopération et de communication, de sorte que les projets peuvent être installés d'une manière adaptée aux réalités locales et susciter le consensus plutôt que les dissensions.

\subsection{Le modèle de la coopérative éolienne}

Le modèle coopératif propose aux citoyens de devenir eux-mêmes propriétaires des unités de génération installées dans leur commune en échange d'un apport en capital sous forme de parts sociales. Si la coopérative est agréée par le Conseil national de la coopération, elle peut lancer un appel à l'épargne publique, qui facilite grandement la récolte de fonds. Selon les normes en vigueur, la coopérative doit supporter au moins 20 \% du coût d'investissement du projet éolien par fonds propres afin de répondre aux exigences des banques, le solde pouvant être couvert par emprunt bancaire. La participation peut revêtir différentes formes. Dans certains cas, la coopérative détient la totalité du projet éolien. Toutefois, la participation citoyenne est le plus souvent minoritaire et s'inscrit dans un partenariat avec un développeur privé.

Les droits de la propriété coopérative sont associés à deux caractéristiques fondamentales. Premièrement, les parts de capital donnent droit à des dividendes versés régulièrement et les citoyens deviennent ainsi bénéficiaires directs des retombées économiques de la production d'énergie. Ensuite, les parts coopératives confèrent aux titulaires le droit de participer aux processus de prises de décision en leur octroyant une voix au sein de l'assemblée générale de l'organisation. En outre, ces deux caractéristiques sont opérées, au sein du modèle coopératif, selon des modalités particulières. Ainsi, le dividende distribué aux associés est généralement limité et les décisions sont prises démocratiquement, selon la règle « une personne - une voix ». Par ailleurs, la littérature met en évidence le niveau de confiance élevé dont jouissent les coopératives grâce à ces spécificités organisationnelles. En effet, elles sont supposées être moins enclines que les entreprises classiques à adopter des comportements opportunistes liés aux asymétries d'information (Hansmann, 1996). Cela leur confère un avantage compétitif pour la production de biens et services caractérisés par un degré élevé de confiance et de qualité non observable.

Les caractéristiques de ces organisations semblent donc répondre aux aspirations de justice et de confiance qui influencent l'acceptabilité des projets d'implantation de parcs éoliens. II importe, cependant, de confronter ces hypothèses normatives à la réalité des données empiriques. Ce dernier point est l'objet de 
la section suivante qui, à partir de données collectées en Flandre, évalue économétriquement l'effet de la propriété coopérative sur l'acceptabilité sociale de l'éolien terrestre.

\section{L'EFFET DE LA PROPRIÉTÉ COOPÉRATIVE SUR L'ACCEPTABILITÉ SOCIALE DE L'ÉOLIEN}

\subsection{Collecte des données}

Les données utilisées ont été récoltées grâce à une enquête en ligne menée de mai à juin 2014 auprès de membres de BeauVent, une coopérative située en Flandre occidentale, et un groupe contrôle d'individus non-membres. Le questionnaire a été conçu pour collecter des données concernant les variables dépendantes et les variables de contrôle pertinentes. Le tableau 1 présente quelques caractéristiques générales de la coopérative.

Tableau 1. Caractéristiques générales de la coopérative en 2013

\begin{tabular}{lc}
\hline Année de création & 2000 \\
Nombre d'équivalents temps plein & 5,37 \\
Nombre de membres & 2391 \\
Capital total (euros) & 4781500 \\
Nombre moyen de parts/membre & 8,00 \\
Période moyenne d'ancienneté des membres (années) & 4,88 \\
\hline
\end{tabular}

Source : créé par l'auteur sur base d'informations fournies par la coopérative.

Une version en ligne du questionnaire a été envoyée aux 849 membres dont l'adresse e-mail était connue de la coopérative. En outre, 43 versions papier du questionnaire ont été distribuées lors d'une assemblée générale. Au total, 222 questionnaires complétés ont été récoltés, ce qui représente un taux de réponse de $25 \%$. La représentativité de cet échantillon a été améliorée en assignant une pondération aux individus afin de reproduire la distribution de certaines variables connues pour l'ensemble de la population : la localisation géographique, le nombre de parts/membre et la période d'ancienneté. D'autre part, les mêmes données ont été récoltées auprès d'un groupe de 502 individus qui n'appartiennent pas à une coopérative. Cette collecte de données a été effectuée par l'Institut de sondage IPSOS, qui dispose d'un panel représentatif de répondants en Flandre. Des quotas ont été imposés de manière que ce groupe contrôle présente des caractéristiques identiques au groupe de référence en termes de sexe, de localisation géographique et de niveau d'éducation. L'objectif recherché était d'obtenir deux groupes qui diffèrent uniquement par leur appartenance ou non à une coopérative. Comme le montre le tableau 2, les deux groupes sont socio-démographiquement proches. 
Trois variables dépendantes ont été considérées. Les deux premières capturent respectivement l'attitude vis-à-vis de l'énergie renouvelable en général et l'attitude envers l'éolien terrestre. La troisième variable dépendante capture la réaction des individus à l'installation d'une éolienne dans leur voisinage immédiat. Trois indicateurs ont été utilisés pour mesurer l'attitude envers l'éolien terrestre et ont ensuite été agrégés en un score unique (alpha de Cronbach $=0.73$ ). Chacune des deux autres attitudes a été mesurée à l'aide d'un seul indicateur. Les réponses devaient être données sur des échelles en cinq points. Le tableau A1 en annexe présente les formulations précises, après traduction du néerlandais, des questions soumises aux répondants.

\subsection{Méthode d'estimation}

Une fois qu'une personne est membre d'une coopérative, il est impossible d'observer ses attitudes dans le cas fictif où elle n'aurait pas rejoint l'organisation. La technique du matching est donc ici utilisée pour résoudre ce problème et pouvoir estimer l'effet moyen de la participation à la coopérative sur les attitudes vis-à-vis de l'éolien terrestre parmi les membres. L'objectif sous-jacent à cette méthode est de construire ex post un groupe contrefactuel identique au groupe de coopérateurs en termes de variables de contrôle (également appelées covariables) susceptibles d'affecter à la fois les attitudes et la sélection dans la coopérative. La méthode du matching associe chaque coopérateur à un individu du groupe contrôle qui présente les mêmes caractéristiques observables, de telle sorte que la participation à la coopérative constitue l'unique différence entre les deux groupes. De cette manière, cette technique reproduit les conditions de l'affectation aléatoire du traitement dans un contexte non expérimental.

Il est toutefois malaisé de trouver des paires d'individus présentant des valeurs identiques pour toutes les covariables. Cette difficulté peut être surmontée grâce à la technique du matching sur le score de propension. Celle-ci consiste à remplacer l'ensemble des covariables par une variable unique, le score de propension, qui désigne, dans le contexte de l'étude, la probabilité d'appartenir à la coopérative conditionnellement aux variables observées ${ }^{3}$. Chaque coopérateur est ainsi apparié à l'individu du groupe contrôle ayant des scores de propension proches. II existe cependant plusieurs critères d'appariement. Afin de nous assurer que les résultats ne dépendent pas du choix d'un critère particulier, nous en avons utilisé deux : le matching kernel sur le score de propension et le matching par plus proche voisin avec remplacement ${ }^{4}$. Nous avons, en outre, limité notre analyse aux individus dont le score appartient au support commun de la distribution des scores des deux groupes.

3. Le score de propension est estimé à l'aide d'un modèle Logit.

4. Le matching kernel sur le score de propension associe à chaque coopérateur une moyenne pondérée de plusieurs individus du groupe contrôle, dont le poids est défini par leur distance au coopérateur. Le matching par plus proche voisin avec remplacement apparie chaque coopérateur à l'individu du groupe contrôle le plus proche, mais un individu du groupe contrôle dont le score de propension est proche de celui de plusieurs membres peut être utilisé à de multiples reprises. 


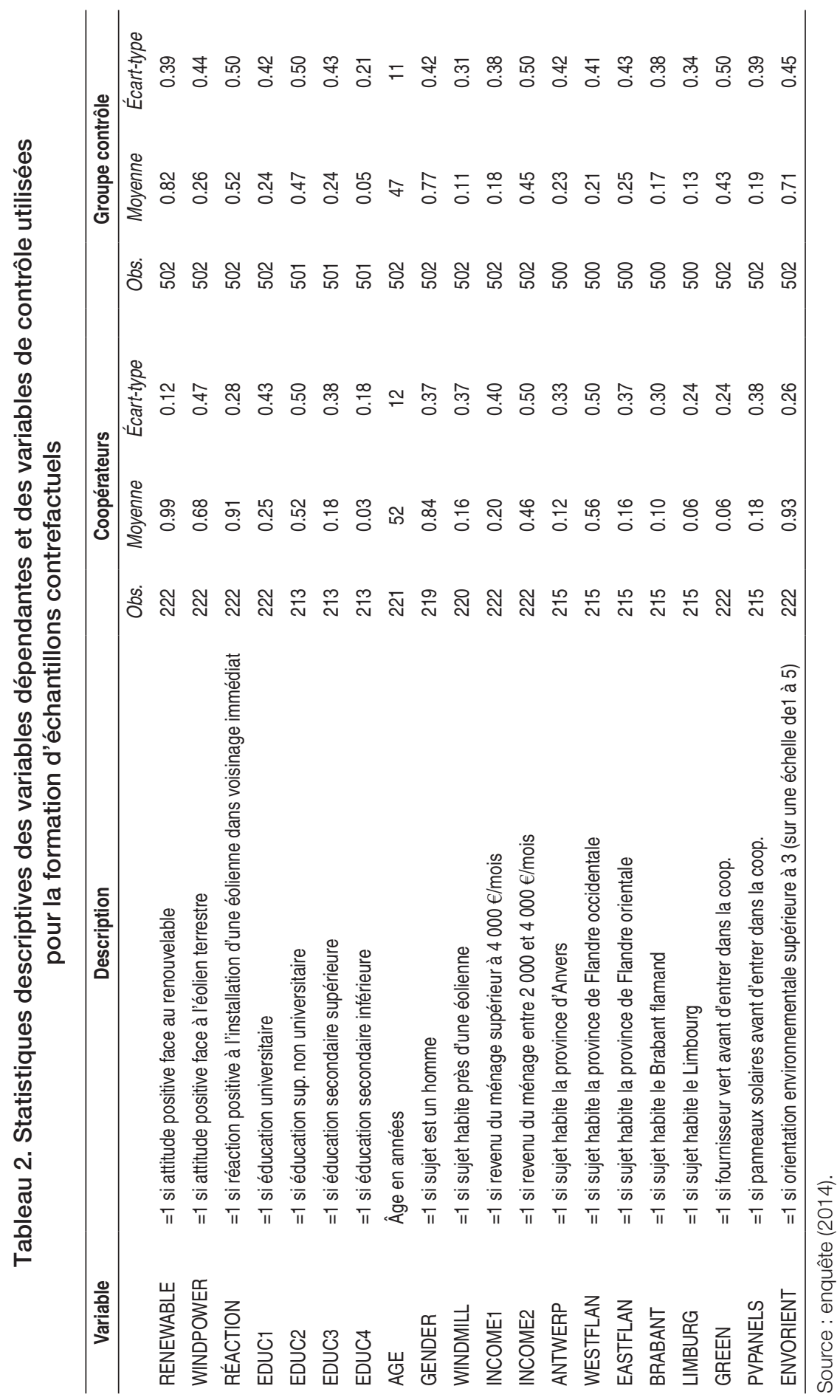


La validité du matching sur le score de propension repose sur l'hypothèse d'indépendance conditionnelle $(\mathrm{HIC})$, selon laquelle la participation à la coopérative est indépendante des effets sur les attitudes étant données les covariables. Un estimateur non biaisé requiert donc que toutes les variables corrélées à la fois à la participation à la coopérative et aux variables dépendantes soient incluses dans les variables de contrôle. Dans le cas présent, la conscience environnementale des individus est susceptible d'affecter simultanément la décision de rejoindre la coopérative et leurs attitudes à l'égard de l'éolien. Afin de corriger ce biais de sélection, nous avons procédé de deux manières. D'une part, nous avons récolté deux variables prétraitement - c'est-à-dire, avant que les individus ne soient membres de la coopérative - pertinentes : la présence de panneaux solaires et le caractère « vert » du fournisseur d'électricité. II a ainsi été demandé aux coopérateurs s'ils possédaient des panneaux solaires avant de rejoindre la coopérative, quel était leur fournisseur d'électricité à l'époque et si celui-ci fournit majoritairement de l'électricité verte ou non. Pour le groupe contrôle, les mêmes données ont été récoltées concernant leur situation actuelle. D'autre part, nous avons construit un index qui capture l'orientation environnementale des participants, basé sur deux dimensions : le degré auquel ils se considèrent eux-mêmes concernés par des préoccupations environnementales (l'identité de soi pro-écologique) et les comportements écologiques qu'ils adoptent au quotidien. Les statistiques descriptives des variables dépendantes et de contrôle utilisées dans l'analyse sont présentées dans le tableau 2.

\subsection{Résultats}

Le tableau 3 présente les différentes estimations. L'effet de la participation à la coopérative sur la première variable dépendante, l'attitude vis-à-vis de l'énergie renouvelable en général, est limité et faiblement significatif pour le matching par plus proche voisin avec remplacement. En revanche, rejoindre une coopérative éolienne augmente significativement l'attitude vis-à-vis de l'éolien terrestre et la réaction de soutien apportée aux éoliennes implantées localement d'environ 0,350 (sur une échelle allant de 1 à 5). Des résultats très similaires sont obtenus quelle que soit la méthode de matching.

Afin de tester la robustesse de ces résultats à d'éventuelles violations de I'HIC, nous avons procédé à une analyse de sensitivité (Becker et Caliendo, 2007). En effet, si les membres de la coopérative diffèrent des non-membres qui leur sont appariés du point de vue d'une variable non observée qui affecte l'attitude à l'égard de l'éolien, l'hypothèse de l'HIC ne sera pas vérifiée et nos estimations seront biaisées. L'analyse de sensitivité consiste à déterminer la force que l'effet d'une variable non observée doit présenter pour infirmer les résultats de la présente analyse de matching. Cette analyse montre que l'effet de la participation à la coopérative sur les deux dernières variables dépendantes demeure significativement positif même en présence d'un biais non observé important ${ }^{5}$. Nous pouvons donc être confiants dans la robustesse des résultats à la présence de biais non observés.

5. Le développement entier et les résultats de cette analyse sont disponibles sur demande. 
Tableau 3. Estimations de l'effet moyen de la participation à la coopérative parmi les membres ( $p$-valeurs entre parenthèses)

\begin{tabular}{lccc}
\hline & $\begin{array}{c}\text { Attitude face à } \\
\text { l'énergie renouvelable }\end{array}$ & $\begin{array}{c}\text { Attitude face } \\
\text { à l'éolien terrestre }\end{array}$ & $\begin{array}{c}\text { Réaction à l'installation } \\
\text { d'une éolienne dans } \\
\text { le voisinage immédiat }\end{array}$ \\
\hline $\begin{array}{l}\text { Matching kernel sur le } \\
\text { score de propension }\end{array}$ & $0.104^{* \star *}(0.004)$ & $0.363^{\star \star *}(0.000)$ & $0.358^{\star \star *}(0.000)$ \\
$\begin{array}{l}\text { Matching par plus proche } \\
\text { voisin avec remplacement }\end{array}$ & $0.082^{\star}(0.061)$ & $0.308^{\star \star \star}(0.000)$ & $0.344^{\star \star *}(0.000)$ \\
$\begin{array}{l}\text { Observations } \\
\text { Coopérateurs }\end{array}$ & 700 & 700 & 700 \\
$\begin{array}{l}\text { Groupe contrôle } \\
\text { Support commun }\end{array}$ & 201 & 201 & 201 \\
Hors support & 499 & 499 & 499 \\
\hline
\end{tabular}

Note : * et ${ }^{\star \star \star}$ indiquent une significativité aux seuils de $10 \%$ et de $1 \%$ respectivement.

\section{CONCLUSION}

L'éolien terrestre, en dépit de la contribution majeure qu'il peut apporter à l'expansion de la capacité d'énergie renouvelable installée en Belgique, souffre d'une acceptabilité sociale limitée, particulièrement au Sud du pays. La propriété coopérative des éoliennes présente des caractéristiques susceptibles de faciliter cette acceptabilité sociale, telles qu'une distribution juste des coûts et des bénéfices liés aux projets, une implication des citoyens dans les processus de décision et l'instauration d'une relation de confiance entre le développeur et les résidents. L'objet de cet article était de tester la validité empirique de cette assertion. Les résultats confirment que la participation à une coopérative augmente significativement l'attitude positive à l'égard de cette technologie. Ces résultats suggèrent que la mise en place d'un contexte institutionnel favorable à cette forme de propriété et le soutien actif à une réelle participation citoyenne pourraient largement contribuer au développement du secteur éolien.

\section{RÉFÉRENCES}

APERE (2014), «Éolien : 23 MW installés en Wallonie en 2014 », disponible sur http:// www.apere.org/doc/140709_CP_APERe_stat_eolien.pdf.

APERE (2015), «Éolien participatif en Wallonie », disponible sur http://www.eolien.be/ Statistiques\%20Participatif.

BeCkER, Sascha O. \& CAliendo, Marco (2007), « Sensitivity analysis for average treatment effects ", Stata Journal, 7 (1), pp. 71-83.

Ben-Ner, Avner \& Putterman, Louis G. (1999), Economics, Values, and Organization. New York: Cambridge University Press. 
CASS, Noel \& WALKER, Gordon (2009), « Emotion and rationality: The characterisation and evaluation of opposition to renewable energy projets ", Emotion, Space and Society, 2 (1), pp. 62-69.

DEVINE-Wright, Patrick (2005), « Beyond NIMBYism: towards an integrated framework for understanding public perceptions of wind energy ", Wind Energy, 8 (2), pp. 125-39.

DeVINE-WriGht, Patrick (2009), " Rethinking NIMBYism: The role of place attachment and place identity in explaining place-protective action ", Journal of Community \& Applied Social Psychology, 19 (6), pp. 426-41.

Frey, Bruno S. \& Oberholzer-Gee, Felix (1997), « The Cost of Price Incentives: An Empirical Analysis of Motivation Crowding-Out », The American Economic Review, 87 (4), pp. 746-55.

Hansmann, Henry (1996), The ownership of enterprise. Cambridge, MA: The Belknap Press of Harvard University Press.

IPSOS-Belgium (2010), « Perception de l'énergie éolienne en Wallonie », Waterloo : Service Public de Wallonie-EDORA.

Olson, Mancur (1965), The Logic of Collective Action: Public Goods and the Theory of Groups. Cambridge, MA: Harvard University Press.

Pepermans, Yves \& Loots, Ilse (2013), « Wind farm struggles in Flanders fields: A sociological perspective ", Energy Policy, 59, pp. 321-28.

WoLSINK, Maarten (2006), "Invalid theory impedes our understanding: a critic on the persistence of the language of NIMBY ", Transactions of the Institute of British Geographers, 31 (1), pp. 85-91.

\section{ANNEXES}

\section{Tableau A1. Indicateurs utilisés pour la mesure des différentes attitudes}

\begin{tabular}{|c|c|}
\hline Attitude concernée & Indicateurs \\
\hline $\begin{array}{l}\text { Attitude vis-à-vis de l'énergie } \\
\text { renouvelable }\end{array}$ & $\begin{array}{l}\text { "Davantage de projets d'énergie renouvelable devraient être développés " } \\
\text { ( } 1 \text { = pas du tout d'accord, } 2 \text { = plutôt pas d'accord, } 3=\text { ni d'accord, ni pas } \\
\text { d'accord, } 4 \text { = plutôt d'accord, } 5 \text { = tout à fait d'accord). }\end{array}$ \\
\hline $\begin{array}{l}\text { Attitude vis-à-vis de l'éolien } \\
\text { terrestre }\end{array}$ & $\begin{array}{l}\text { “Davantage d'éoliennes terrestres devraient être installées » } \\
\text { "Les éoliennes sont dommageables pour le paysage » } \\
\text { "Les éoliennes offrent une réponse à la problématique climatique » }\end{array}$ \\
\hline $\begin{array}{l}\text { Réaction à l'installation d'une } \\
\text { éolienne dans le voisinage } \\
\text { immédiat }\end{array}$ & $\begin{array}{l}\text { "Si une éolienne était construite dans votre environnement immédiat } \\
\text { (moins de } 5 \mathrm{~km} \text { de votre habitat), votre réaction serait... " } \\
\text { ( } 1 \text { = forte opposition, } 2=\text { opposition modérée, } 3=\text { ni opposition, ni soutien, } \\
4 \text { = soutien modéré, } 5=\text { soutien fort). }\end{array}$ \\
\hline
\end{tabular}

Source : créé par l'auteur. 